\title{
Bar weights of bar partitions and spin character zeros
}

\author{
Christine Bessenrodt
}

Received: 18 July 2006 / Accepted: 21 November 2006 /

Published online: 10 January 2007

(C) Springer Science + Business Media, LLC 2007

\begin{abstract}
The main combinatorial result in this article is a classification of bar partitions of $n$ which are of maximal $p$-bar weight for all odd primes $p \leq n$. As a consequence, we show that apart from very few exceptions any irreducible spin character of the double covers of the symmetric and alternating groups vanishes on some element of odd prime order.
\end{abstract}

Keywords Bar partitions · Bar cores · Bar weights · Symmetric group · Alternating group $\cdot$ Spin characters $\cdot$ Character zeros

Mathematics Subject Classification 05A17, 20C30

\section{Introduction}

A well known result by Burnside states that any non-linear irreducible character of a finite group vanishes on some element of the group. This was refined in [9], where it was shown that such a character always has a zero at an element of prime power order; it had also been noticed in [9] that any non-linear irreducible character of a finite simple group except possibly the alternating groups even vanishes on some element of prime order. In [5] it was then shown that this character property also holds for the alternating and the symmetric groups. Indeed, this vanishing property was a consequence of a combinatorial result on the weights of partitions.

An extended abstract for this paper appeared in the Proceedings of the FPSAC'06 conference.

C. Bessenrodt $(\square)$

Institut für Algebra, Zahlentheorie und Diskrete Mathematik, Fakultät für Mathematik und Physik, Leibniz Universität Hannover, Welfengarten 1, D-30167 Hannover, Germany

e-mail: bessen@math.uni-hannover.de 
Here, we prove a corresponding vanishing property for irreducible spin characters of the double covers of the symmetric and alternating groups on elements of odd prime order; this is obtained from a result on bar weights of partitions into distinct parts.

The elements of odd prime order $p$ which we are going to use in the double cover $\tilde{S}_{n}$ of the symmetric group $S_{n}$ are those of maximal $p$-bar weight, i.e., the corresponding cycle type has $\left\lfloor\frac{n}{p}\right\rfloor$ parts of size $p$. (Here $\lfloor\cdot\rfloor$ denotes the floor function. Thus $\lfloor x\rfloor$ is the integral part of $x \in \mathbb{R}$.)

For $n \in \mathbb{N}$, we denote by $D(n)$ the set of partitions of $n$ into distinct parts, and we set $D=\cup_{n} D(n)$. Now consider a partition $\lambda \in D(n)$, also called a bar partition. For a given integer $r \in \mathbb{N}$, we denote by $\bar{w}_{r}(\lambda)$ the $r$-bar weight of $\lambda$, i.e., $\bar{w}_{r}(\lambda)$ is the maximal number of $r$-bars that can successively be removed from $\lambda$. The resulting bar partition after removing this maximal number of $r$-bars is then the $r$-bar core (or $\bar{r}$-core) $\lambda_{(\bar{r})}$ of $\lambda$ (see [7] or [12] for details). In this paper, we will only deal with the case where $r$ is a prime number $p$. In the main result we classify the bar partitions of $n$ which have maximal $\bar{p}$-weight $\left\lfloor\frac{n}{p}\right\rfloor$ for all odd primes $p \leq n$.

We denote by $\mathcal{O}(n)$ the set of partitions of $n$ into odd parts; elements of $\tilde{S}_{n}$ whose image in $S_{n}$ has cycle type in $\mathcal{O}(n)$ are said to be of odd type (or of type $\mathcal{O}$ ). We now describe the connection to the vanishing of spin characters on elements of odd type. The irreducible spin characters of $\tilde{S}_{n}$ are labelled by the bar partitions $\lambda$ of $n$ (and signs). The recursion formula given by Morris [10] for spin character values on elements of odd type in $\tilde{S}_{n}$ shows that an irreducible spin character labelled by $\lambda$ vanishes on a $p$-element of maximal weight (where $p$ is odd), if the $\bar{p}$-weight of $\lambda$ is not maximal (or equivalently, the $\bar{p}$-core of $\lambda$ is not small, i.e., has size at least $p$ ). Note that the spin character values occur as factors in the coefficients of the expansion of Schur $Q$-functions $Q_{\lambda}$ into power sum functions $p_{\alpha}$, where $\lambda \in D(n)$ and $\alpha \in \mathcal{O}(n)$, so the vanishing of the spin character values is connected with the support of this expansion.

For $p=2$, suitable notions to consider are the $\overline{4}$-weight and the $\overline{4}$-core of $\lambda$, respectively, which are computed using the $\overline{4}$-abacus with one runner for the even parts, and two conjugate runners for the parts $\equiv 1,3 \bmod 4$; this fits with the distribution of spin characters into the 2-blocks of $\tilde{S}_{n}$ (see [3]).

Our main result is the following:

Theorem 1.1. Let $\lambda$ be a bar partition of $n \in \mathbb{N}$. Then the following holds: $\bar{w}_{p}(\lambda)=$ $\left\lfloor\frac{n}{p}\right\rfloor$ for all odd primes $p \leq n$ if and only if $\lambda=(n)$ or $\lambda=(n-1,1)$, where $n=2^{a}+2$ for some $a \in \mathbb{N}$, or one of the following occurs:

$$
\begin{aligned}
& n=5: \lambda=(3,2) \\
& n=6: \lambda=(3,2,1) \\
& n=8: \lambda=(5,2,1) \\
& n=9: \lambda=(4,3,2) \\
& n=10: \lambda=(4,3,2,1) \text { or }(7,3)
\end{aligned}
$$

If, in addition, also the $\overline{4}$-core of $\lambda$ is small (i.e., it is of size smaller than 2), then $\lambda=(n)$ with $n \not \equiv 3 \bmod 4$, or $\lambda$ is one of $(3,1),(3,2,1),(4,3,2,1)$. 
The combinatorial classification result has the desired consequence for the spin character zeros; before stating this, first we have to introduce some more notation.

A partition $\lambda \in D(n)$ is in $D^{+}(n)$ (or $D^{-}(n)$, resp.) if $n-l(\lambda)$ is even (or odd, resp.). We denote by $\langle\mu\rangle$ the irreducible spin character of $\tilde{S}_{n}$ corresponding to $\mu \in D^{+}(n)$, and by $\langle\mu\rangle_{+},\langle\mu\rangle_{-}=\operatorname{sgn} \cdot\langle\mu\rangle_{+}$, the irreducible spin characters of $\tilde{S}_{n}$ associated to $\mu \in D^{-}(n)$. Furthermore, we let $\langle\langle\mu\rangle\rangle$ denote the irreducible spin character of $\tilde{A}_{n}$ corresponding to $\mu \in D^{-}(n)$ (which is the reduction of $\langle\mu\rangle_{ \pm}$), and $\langle\langle\mu\rangle\rangle_{ \pm}$the irreducible spin characters of $\tilde{A}_{n}$ associated to $\mu \in D^{+}(n)$ (which are conjugate and sum to the reduction of $\langle\mu\rangle$, and which differ only on the critical classes of cycle type $\left.\lambda \in D^{+}\right)$.

We refer to [7] for further details on the irreducible spin characters of $\tilde{A}_{n}$.

A special rôle is played by the basic spin characters of $\tilde{S}_{n}$ and $\tilde{A}_{n}$; these are the spin characters labelled by the bar partition $(n)$, i.e., $\langle n\rangle_{( \pm)}$and $\langle\langle n\rangle\rangle_{( \pm)}$. On an element of type $\alpha \in \mathcal{O}(n)$ the basic spin character $\langle n\rangle_{( \pm)}$has the value $2^{\left[\frac{l(\alpha)}{2}\right]}$ (similarly for the basic spin characters of $\tilde{A}_{n}$ ); hence the basic spin characters do not have any zeros on elements of odd type. We will thus only consider non-basic spin characters.

Theorem 1.2. Let $n \in \mathbb{N}, n \geq 4$. Let $\chi$ be any non-basic irreducible spin character of a double cover $\tilde{S}_{n}$ of the symmetric group $S_{n}$ or a double cover $\tilde{A}_{n}$ of the alternating group $A_{n}$. Then $\chi$ vanishes on some element of odd prime order, except if $\chi$ is labelled by $(n-1,1)$ with $n=2^{a}+2$ for some $a \in \mathbb{N}$, or by one of the partitions $(3,2)$, $(3,2,1)$ or $(5,2,1)$.

Proof: For the spin characters of $\tilde{S}_{n}$, this follows from Theorem 1.1, after checking that the spin characters for the other exceptional partition labels for small $n$, i.e., $(4,3,2),(4,3,2,1),(7,3)$, indeed have zeros of odd prime order; in fact, they vanish on elements of cycle type $\left(3^{2}, 1^{*}\right)$. For $(3,2),(3,2,1)$ or $(5,2,1)$, the corresponding spin characters do not have a zero of odd prime order. Also, the spin character to $\left(2^{a}+1,1\right)$ cannot vanish on an element of cycle type $\left(p^{m}, 1^{r}\right)$ for an odd prime $p$, as there is exactly one way of removing $p$-bars and one can always remove $m p$-bars.

For the spin characters of $\tilde{A}_{n}$, we now discuss how to deduce the result from the one above. Let $\lambda \in D(n)$, not one of the exceptional partitions in Theorem 1.1.

If $\lambda \in D^{-}(n)$, then $\langle\lambda\rangle_{ \pm}\left(\sigma_{\alpha}\right)=0$ for some $\alpha \in \mathcal{O}(n)$ with $\sigma_{\alpha}$ of odd prime order. Then $\sigma_{\alpha} \in \tilde{A}_{n}$ and also $\langle\langle\lambda\rangle\rangle\left(\sigma_{\alpha}\right)=0$.

If $\lambda \in D^{+}(n)$, then $\langle\lambda\rangle\left(\sigma_{\alpha}\right)=0$ for some $\alpha \in \mathcal{O}(n)$ with $\sigma_{\alpha}$ of odd prime order; again, $\sigma_{\alpha} \in \tilde{A}_{n}$. If $\lambda \in\left(D^{+} \cap \mathcal{O}\right)(n)$, then the critical class $\sigma_{\lambda}$ for $\langle\langle\lambda\rangle\rangle_{ \pm}$is in $\mathcal{O}(n)$, but $\langle\lambda\rangle\left(\sigma_{\lambda}\right)= \pm 1$, thus $\lambda \neq \alpha$ and then also the spin characters $\langle\langle\lambda\rangle\rangle_{ \pm}$vanish on $\sigma_{\alpha}$. If $\lambda \in D^{+}(n) \backslash\left(D^{+} \cap \mathcal{O}\right)(n)$, then the critical class $\sigma_{\lambda}$ for $\langle\langle\lambda\rangle\rangle_{ \pm}$is not in $\mathcal{O}(n)$, so again $\lambda \neq \alpha$ and the spin characters $\langle\langle\lambda\rangle\rangle_{ \pm}$vanish on $\sigma_{\alpha}$.

Remark 1.3. (i) If an irreducible character $\chi$ of a finite group $G$ has a zero at an element of prime order $p$, then $p$ divides $\chi(1)$. Note that the irreducible spin characters of $\tilde{S}_{n}$ and $\tilde{A}_{n}$ of prime power degree have been classified in [4]; from Theorem 1.2 we can immediately recover the classification of irreducible spin characters of 2-power degree for these groups. In fact, here they are exactly those that do not have a zero at an element of odd prime order. 
The converse of the statement above does not hold, even for $G=\tilde{S}_{n}$. The spin character $\langle 8,4\rangle$ is of degree $5280=2^{4} \cdot 3 \cdot 5 \cdot 11$, but the character does not vanish on any element of order 3 .

(ii) Any irreducible spin character of $\tilde{S}_{n}$ vanishes on the classes which are not of type $\mathcal{O}(n)$ or $D^{-}(n)$ as these do not split in $\tilde{S}_{n}$; in particular, for all $n \geq 4$, these characters do always have (trivial) zeros at all elements of order 2 . The even elements of order 2 are also zeros of the irreducible spin characters of $\tilde{A}_{n}$.

Note that there is a simple relation between the $\bar{p}$-weight of a bar partition $\lambda$ and the defect of the spin $p$-block containing the irreducible spin character(s) of $S_{n}$ or $A_{n}$ labelled by $\lambda$ (see [12]). For a prime $p \leq n$, the basic spin character(s) of $\tilde{S}_{n}$ or $\tilde{A}_{n}$ are contained in one $p$-block which we call the basic $p$-block of $\tilde{S}_{n}$ or $\tilde{A}_{n}$, respectively. The following is then another direct consequence of Theorem 1.1 (note that for $a>2$ the spin character to $\left(2^{a}+1,1\right)$ is not in the basic $p$-block for any odd prime $p$ not dividing $n$ and $n-1)$.

Corollary 1.4. Let $n \in \mathbb{N}, n \geq 4$.

(i) The basic spin characters $\langle n\rangle_{( \pm)}$, the spin characters $\langle n-1,1\rangle$ where $n=2^{a}+2$ for some $a \in \mathbb{N}$, and the spin characters $\langle 3,2\rangle_{ \pm},\langle 3,2,1\rangle_{ \pm},\langle 5,2,1\rangle_{ \pm}$, $\langle 4,3,2\rangle,\langle 4,3,2,1\rangle,\langle 7,3\rangle$ are the only irreducible spin characters of $\tilde{S}_{n}$ which are in spin p-blocks of maximal defect for all odd primes $p$.

The spin characters $\langle 3,1\rangle,\langle 5,1\rangle,\langle 3,2\rangle_{ \pm},\langle 3,2,1\rangle_{ \pm},\langle 4,3,2\rangle,\langle 7,3\rangle$ are the only non-basic spin characters contained in the basic p-block for all odd primes $p \leq n$.

(ii) The basic spin characters $\langle\langle n\rangle\rangle_{( \pm)}$, the spin characters $\langle\langle n-1,1\rangle\rangle_{ \pm}$where $n=$ $2^{a}+2$ for some $a \in \mathbb{N}$, and the spin characters $\langle\langle 3,2\rangle\rangle,\langle\langle 3,2,1\rangle\rangle,\langle\langle 5,2,1\rangle\rangle$, $\langle\langle 4,3,2\rangle\rangle_{ \pm},\langle\langle 4,3,2,1\rangle\rangle_{ \pm},\langle\langle 7,3\rangle\rangle_{ \pm}$are the only irreducible spin characters of $\tilde{A}_{n}$ which are in spin p-blocks of maximal defect for all odd primes $p$.

The spin characters $\langle\langle 3,1\rangle\rangle_{ \pm},\langle\langle 5,1\rangle\rangle_{ \pm},\langle\langle 3,2\rangle\rangle,\langle\langle 3,2,1\rangle\rangle,\langle\langle 4,3,2\rangle\rangle_{ \pm}$, $\langle\langle 7,3\rangle\rangle_{ \pm}$are the only non-basic spin characters contained in the basic p-block of $\tilde{A}_{n}$ for all odd primes $p \leq n$.

As mentioned above, the 2-block distribution of the spin characters was determined in [3]. Employing this, we immediately obtain the following result for $\tilde{S}_{n}$ (a corresponding result also holds for $\tilde{A}_{n}$ ):

Corollary 1.5. Let $n \in \mathbb{N}, n \geq 4$.

(i) The basic spin characters $\langle n\rangle_{( \pm)}$for $n \not \equiv 3 \bmod 4$ and the spin characters $\langle 3,1\rangle$, $\langle 3,2,1\rangle_{ \pm},\langle 4,3,2,1\rangle$ are the only irreducible spin characters of $\tilde{S}_{n}$ which are in p-blocks of maximal defect for all primes $p$.

(ii) The spin characters $\langle 3,1\rangle$ and $\langle 3,2,1\rangle_{ \pm}$are the only non-basic spin characters contained in the basic $p$-block for all primes $p \leq n$.

Note that the statements on the basic blocks in the second part of Corollary 1.4(i) and in Corollary 1.5(ii) were also obtained in [2]. 
The paper is organized as follows. Section 2 contains some results on bar lengths. In Section 3 we consider bar partitions of maximal bar weight. We will use suitable algorithms to generate "large" first row bar lengths in the bar partitions under consideration. The final section contains the proof of the main result.

\section{Bar lengths of bar partitions}

We refer to $[7,8,12]$ for details about partitions, Young diagrams, hooks and bar partitions, shifted diagrams and bars, respectively.

Consider a partition $\lambda=\left(\lambda_{1}, \lambda_{2}, \ldots, \lambda_{l}\right)$ of the integer $n$. Thus $\lambda_{1} \geq \lambda_{2} \geq \cdots \geq$ $\lambda_{l}>0$ and $\lambda_{1}+\lambda_{2}+\cdots+\lambda_{l}=n$, with integer parts $\lambda_{i} ; l=l(\lambda)$ is the length of $\lambda$. The Young diagram of $\lambda$ consists of $n$ boxes with $\lambda_{i}$ boxes in the $i$ th row. We refer to the boxes in matrix notation, i.e. the $(i, j)$-box is the $j$ th box in the $i$ th row. The $(i, j)$-hook consists of the boxes in the Young diagram to the right of and below the $(i, j)$-box, and including this box. The number of boxes in this hook is its hook length, denoted by $h_{i j}$.

For $\lambda \in D$, we consider the corresponding shifted diagram, where in the $i$ th row we start on the diagonal at $(i, i)$ rather than at the box $(i, 1)$. By flipping this over the diagonal and then gluing it to the shifted diagram of $\lambda$, we obtain the shift symmetric diagram $S(\lambda)$ on $2 n$ boxes. The bar lengths in $\lambda$ correspond to the hook lengths in the $\lambda$-boxes of $S(\lambda)$; the bar length at position $(i, j)$ is then denoted $b_{i j}$; we abbreviate the bar lengths in the first row by $b_{1 i}=b_{i}$, or also by $b_{i}^{\lambda}$ when necessary.

Furthermore, we denote by $\bar{h}_{\lambda}$ the product of all the bar lengths of $\lambda$.

Example. Take $\lambda=(4,3,1)$. In the shift symmetric diagram below the bar lengths are filled into the corresponding boxes of $\lambda$.

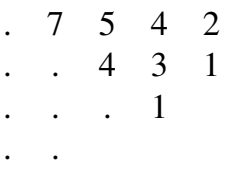

Thus, $\bar{h}_{\lambda}=7 \cdot 5 \cdot 4^{2} \cdot 3 \cdot 2$.

The removal of a $p$-bar from $\lambda \in D(n)$ corresponds to taking a part $p$ or two parts summing to $p$ out of $\lambda$, or subtracting $p$ from a part of $\lambda$ if possible (i.e., if the resulting partition is in $D(n-p))$. Doing this as long as possible gives the $\bar{p}$-core $\lambda_{(\bar{p})}$ of $\lambda$; the number of $p$-bars removed is then the $p$-bar weight $\bar{w}_{p}(\lambda)$ of $\lambda$ (see [7] or [12] for details). These operations may also be performed on a suitable $\bar{p}$-abacus.

Example. Take $p=3, \lambda=(7,3,2,1)$. Removing a bar of length 3 from $\lambda$ can be achieved by removing the parts 2 and 1 from $\lambda$, or by removing the part 3 , or by replacing 7 by 4 . When we do this in succession, we have reached the bar partition (4), from which we can remove a further 3 -bar and thus obtain $(1)=\lambda_{(\overline{3})}$; the $\overline{3}$-weight of $\lambda$ is 4 .

We will often make use of the following property of the $p$-bar weight of a partition (see $[11,12]$ ); the Lemma may easily be proved by considering the $\bar{p}$-abacus (see [12]). 
Lemma 2.1. Let $p$ be an odd prime. If $\lambda$ is a bar partition of $\bar{p}$-weight $\bar{w}_{p}(\lambda)=w$, then $\lambda$ has exactly $w$ bars of length divisible by $p$. In particular, if $\lambda$ has a bar of length divisible by $p$, then it has a bar of length $p$.

This is used to prove some easy but crucial results about bar lengths (compare this with [4] where a similar Lemma for hook lengths is used).

As mentioned before, for $p=2$, a suitable parameter to consider is the $\overline{4}$-core of $\lambda$ which is computed using the $\overline{4}$-abacus with one runner for the even parts, and two conjugate runners for the parts $\equiv 1,3 \bmod 4$; in contrast to the $\bar{p}$-abacus for odd $p$, here we are allowed to subtract 2 from the even parts (so these will be removed when computing the $\overline{4}$-core).

From now on, $\lambda=\left(\lambda_{1}, \ldots, \lambda_{l}\right)$ is always a bar partition of $n$, of length $l$.

The following easy proposition will often be useful.

Proposition 2.2. Assume that $\bar{w}_{p}(\lambda)=\left\lfloor\frac{n}{p}\right\rfloor$ for the odd prime $p \leq n$.

(i) Let $\mu$ be obtained from $\lambda$ by removing the first row. Then the number of bars in $\mu$ divisible by $p$ is equal to the number of p-multiples $k p, 1 \leq k \leq\left\lfloor\frac{n}{p}\right\rfloor$, which are not first row bar lengths of $\lambda$.

In particular, if $p \nmid \bar{h}_{\mu}$, then $p, 2 p, \ldots,\left\lfloor\frac{n}{p}\right\rfloor p$ are first row bar lengths of $\lambda$.

(ii) If $n-\lambda_{1}<p$, then $p, 2 p, \ldots,\left\lfloor\frac{n}{p}\right\rfloor p$ are first row bar lengths of $\lambda$.

Proof: Parts (i) and (ii) follow immediately from Lemma 2.1.

Note that the first row bar lengths of $\lambda$ can explicitly be given (see [12]); the set of these numbers is

$$
\left\{\lambda_{1}+\lambda_{2}, \ldots, \lambda_{1}+\lambda_{l}\right\} \cup\left\{1, \ldots, \lambda_{1}\right\} \backslash\left\{\lambda_{1}-\lambda_{2}, \ldots \lambda_{1}-\lambda_{l}\right\}
$$

In particular, the largest bar length in $\lambda$ is $\lambda_{1}+\lambda_{2}$ (which also follows easily from the definition).

We also recall a useful result due to Hanson:

Theorem 2.3 ([6]). The product of $k$ consecutive numbers all greater than $k$ contains a prime divisor greater than $\frac{3}{2} k$, with the only exceptions $3 \cdot 4,8 \cdot 9$ and $6 \cdot 7 \cdot 8 \cdot 9 \cdot 10$.

We can now deal with the "bar case" in analogy to dealing with the hook case first in the situation of partitions. We call a bar partition $\lambda$ a $\operatorname{bar}$ if $l(\lambda) \leq 2$.

Proposition 2.4. Let $\lambda=(n-k, k)$ for some $k \in \mathbb{N}_{0}, k<n-k$. Then $\bar{w}_{p}(\lambda)=\left\lfloor\frac{n}{p}\right\rfloor$ for all odd primes $p \leq n$ if and only if one of the following holds:

(i) $k=0$, i.e., $\lambda=(n)$.

(ii) $k=1$ and $n=2^{a}+2$ for some $a \in \mathbb{N}_{0}$, i.e., $\lambda=\left(2^{a}+1,1\right)$.

(iii) $\lambda$ is one of $(3,2),(7,3)$.

If, in addition, also the $\overline{4}$-core of $\lambda$ is small, then $\lambda=(n)$ or $\lambda$ is one of $(2,1),(3,1)$. 
Proof: One easily checks that all the partitions listed in (i)-(iii) have the stated property. For the converse, we may assume $k>0$. The two largest bar lengths in the first row are $b_{1}=n$ and $b_{2}=n-k$. For $k \geq 3$, consider the following product of "missing" first row bar lengths:

$$
\pi=(n-k+1)(n-k+2) \cdots(n-1) .
$$

This has $k-1$ factors, all greater than $n-k>k$, hence by Theorem 2.3 one of these factors has a prime divisor $q>\frac{3}{2}(k-1)$, except if $(n, k)=(10,3)$ (note that the possibilities $(5,3)$ and $(11,6)$ do not occur as we are assuming $k<n-k)$. As $k \geq 3$, we then have such a prime divisor $q>k=n-\lambda_{1}$, except when $(n, k)=(10,3)$, and indeed this gives one of the exceptional partitions listed in (iii). As $\lambda$ is of maximal $\bar{q}$-weight, Proposition 2.2 implies that all multiples of $q$ which are at most $n$ are first row bar lengths in all other partitions with $k \geq 3$, giving a contradiction.

For $k=2$, the partition $\lambda=(3,2)$ is an exception, as listed in (iii). If $\lambda \neq(3,2)$, then $\pi_{1}=n-1$ and $\pi_{2}=n-4$ are missing bar lengths in the first row; note that $n>5$, thus $n-4>1$. Now one of the numbers $\pi_{1}, \pi_{2}$ is odd, hence has a prime divisor $q \geq 3$. But then, using Proposition 2.2 again, we obtain a contradiction.

It remains to consider the case $k=1$. Then only the bar length $n-2$ is missing in the first row; if this has an odd prime divisor, we get a contradiction as before. Hence the only possibility in this case is that $n=2^{a}+2$ for some $a \in \mathbb{N}_{0}$.

If we now assume that in addition the $\overline{4}$-core of $\lambda$ is small, then one easily sees that only the partitions $(n)$ and $(2,1),(3,1)$ remain.

Lemma 2.5. Let $\lambda \in D(n)$. Let $s$ be a bar length of $\lambda$ with $\frac{n}{2} \leq s$. Then $s$ is a first row bar length of $\lambda$ or $s=b_{23}=\lambda_{2}+\lambda_{3}$. In the second case, $b_{1}, b_{2}$ are then the only first row bar lengths $\geq \frac{n}{2}$.

Proof: If $s$ is not a first row bar length, then $s \leq b_{23}=\lambda_{2}+\lambda_{3}$. If $s \neq b_{23}$, then $\frac{n}{2} \leq s \leq \lambda_{2}+\lambda_{4}$. But since $\lambda_{1}+\lambda_{3}>\lambda_{2}+\lambda_{4}$, then $\lambda_{1}+\lambda_{3}+\lambda_{2}+\lambda_{4}>n-$ a contradiction. The final assertion follows using the same inequality.

Corollary 2.6. Let $\lambda \in D(n)$ be of maximal $\bar{p}$-weight for all odd primes $p \leq n$ with $p \geq \frac{n}{2}$. Let $q$ be the smallest prime with $q \geq \frac{n}{2}$. Then any prime $r$ with $q<r \leq n$ is a first row bar length of $\lambda$.

Corollary 2.7. Let $n=13,14$ or $n \geq 17$. Let $\lambda \in D(n)$ be of maximal $\bar{p}$-weight for all odd primes $p$ with $\frac{n}{2} \leq p \leq n$. Then all bar lengths $\geq \frac{n}{2}$ are first row bar lengths of $\lambda$.

Proof: The condition on $n$ guarantees that there are at least three odd primes $p$ with $\frac{n}{2} \leq p \leq n$. All these have to be bar lengths of $\lambda$. If there is a further bar length $s \geq \frac{n}{2}$ in the second row, then we immediately have a contradiction. If some prime $q \geq \frac{n}{2}$ is not a first row bar length, then by Lemma 2.5 we have only two larger bar lengths, thus we are in the situation where there are only three primes, and these have to be the numbers $\lambda_{1}+\lambda_{2}, \lambda_{1}+\lambda_{3}$ and $\lambda_{2}+\lambda_{3}$. But these three numbers can not all be odd, giving a contradiction. Hence all the bar lengths $\geq \frac{n}{2}$ are first row bar lengths. 


\section{Forcing large bar lengths}

We can now use a similar procedure as in [4] to force the bar partitions under investigation to have large bar lengths in their first row:

Proposition 3.1. Let $n \geq 17$. Let $\lambda \in D(n)$ be of maximal $\bar{p}$-weight for all odd primes $p \leq n$. Let $s_{1}<s_{2}<\cdots<s_{r} \leq n$ and $t_{1}<t_{2}<\cdots<t_{r} \leq n$ be sequences of integers satisfying

(i) $s_{i}<t_{i}$ for all $i$;

(ii) $s_{1}, t_{1}$ are primes $>\frac{n}{2}$;

(iii) for $1 \leq i \leq r-1$, each of $s_{i+1}, t_{i+1}$ has a prime divisor exceeding $2 n-s_{i}-t_{i}$.

Then $s_{1}, \ldots, s_{r}, t_{1}, \ldots, t_{r}$ are first row bar lengths of $\lambda$.

Proof: We use induction on $i$. For $s_{1}, t_{1}$ it follows from Corollary 2.7 that they are first row bar lengths, as by (ii) $s_{1}, t_{1}$ are primes $>\frac{n}{2}$. We now assume the assertion up to $i$. Then $s_{i}+t_{i} \leq \lambda_{1}+\lambda_{2}+\lambda_{1}+\lambda_{3} \leq n+\lambda_{1}$. Now let $q$ be a prime divisor of $s_{i+1}$ (or $t_{i+1}$, resp.) as in (iii); then

$$
q>2 n-s_{i}-t_{i} \geq n-\lambda_{1},
$$

and hence by Proposition $2.2 s_{i+1}$ (or $t_{i+1}$, resp.) is a first row bar length of $\lambda$.

Now we can use a similar strategy as in [1]. When we can show that there are sequences as in Proposition 3.1 such that $t_{r}$ comes "close" to $n$, then the largest bar $b_{1}$ of $\lambda$ has length "close" to $n$, and thus $\lambda$ is "almost" a two-part partition. We use a greedy algorithm to check for suitable sequences. Start with two large primes $s_{1}<t_{1}$ close to $n$. Then $2 n-s_{1}-t_{1}$ is small. Choose if possible two integers $s_{2}$ and $t_{2}$ with $s_{2}<t_{2}, s_{1}<s_{2} \leq n, t_{1}<t_{2} \leq n$ each having a prime divisor exceeding $2 n-s_{1}-t_{1}$. Then $2 n-s_{2}-t_{2}<2 n-s_{1}-t_{1}$. Choose if possible two integers $s_{3}$ and $t_{3}$ with $s_{3}<t_{3}, s_{2}<s_{3} \leq n, t_{2}<t_{3} \leq n$ each having a prime divisor exceeding $2 n-s_{2}-t_{2}$, and so on. For any numbers $s_{i}, t_{i}$ in this process, we have $t_{i} \leq b_{1}=$ $\lambda_{1}+\lambda_{2}$ by Proposition 3.1.

Now from [1] we already know that for very large $n$ this algorithm indeed terminates quite close to $n$. To state the precise result, we first recall some notation from [1].

Suppose that $n \geq 3$ is a positive integer. Consider two finite increasing sequences of integers $\left\{A_{i}\right\}$ and $\left\{B_{i}\right\}$ which satisfy the following properties:

(i) $A_{1}<B_{1} \leq n$ are two "large" primes.

(ii) For every $i$, we have $A_{i}<B_{i} \leq n$.

(iii) If $B_{i}<n$, then $A_{i+1}<B_{i+1}$ are integers not exceeding $n$ each with a prime factor exceeding $2 n-A_{i}-B_{i}$.

Then denote by $A(n)$ (resp. $B(n))$ the largest integer in such a sequence $\left\{A_{i}\right\}$ (resp. $\left.\left\{B_{i}\right\}\right)$.

For $n$ in a large range it was already checked (by computer) for the proof of the classification result in [1] that the algorithm ends very close to $n$, more precisely, we have: 
Proposition 3.2. If $29 \leq n \leq 9.25 \cdot 10^{8}$, then there is a pair of sequences $\left\{A_{i}\right\}$ and $\left\{B_{i}\right\}$ as above for which

$$
n-B(n) \leq 4
$$

For sufficiently large $n$ we have the following result from [1]:

Theorem 3.3. If $n>9.25 \cdot 10^{8}$, then there is a pair of sequences $\left\{A_{i}\right\}$ and $\left\{B_{i}\right\}$ as above for which

$$
n-B(n) \leq 225 \text {. }
$$

Using Proposition 3.1 we can thus deduce:

Corollary 3.4. Let $n \in \mathbb{N}$. Let $\lambda \in D(n)$ be of maximal $\bar{p}$-weight for all odd primes $p \leq n, b_{1}=\lambda_{1}+\lambda_{2}$ its largest bar length. Then

(i) For $29 \leq n \leq 9.25 \cdot 10^{8}, n-b_{1} \leq 4$.

(ii) For $n>9.25 \cdot 10^{8}, n-b_{1} \leq 225$.

So we still have the tasks to reduce 225 to some manageable number when $n$ is large, and to deal with the cases where $n-b_{1} \leq 4$ (and $n$ is arbitrary) or where $n-b_{1}$ is bounded by some reasonably sized number (when $n$ is large).

For a positive real number $x$, define $\pi(x)$ by

$$
\pi(x):=\#\{p \text { prime } \mid p \leq x\} .
$$

We need a crucial number-theoretic Lemma for reducing $d=n-b_{1}$ and $k=\lambda_{2}-$ $\lambda_{3}-1$; a similar result was already used in [5] but it has to be adapted for the purposes here.

Lemma 3.5. Let $5 \leq r \leq 1000$. Then any product of $r$ consecutive integers larger than $5.5 \cdot 10^{8}$ has a prime divisor $q>2.15 \cdot r$, when $r \leq 10, q>2.58 \cdot r$, when $11 \leq$ $r \leq 21$, and $q>3 \cdot r$, when $r \geq 22$.

Proof: Let $n \in \mathbb{N}$ with $n>5.5 \cdot 10^{8}$. Assume that all prime factors in the $r$ consecutive numbers $n-r+1, \ldots, n-1, n$ are at most $c \cdot r$, where $c=2.15, c=2.58$ and $c=3$ for the ranges of $r$ as given in the statement above. Then we obtain

$$
\left(\frac{n}{r}\right)^{r}<\left(\begin{array}{l}
n \\
r
\end{array}\right)=\prod_{p \mid\left(\begin{array}{l}
n \\
r
\end{array}\right)} p^{b_{p}} \leq n^{\pi(c r)}
$$

where $p^{b_{p}}$ is the maximal power of $p$ dividing $\left(\begin{array}{l}n \\ r\end{array}\right)$ (this is known to be bounded by $n$ ). Equivalently,

$$
(r-\pi(c r)) \ln (n)<r \ln r .
$$


Note that for $c$ as chosen above, $r>\pi(c r)$ for the corresponding ranges of $r$. Hence, if the assumption holds then

$$
\ln (n)<\frac{r \ln (r)}{r-\pi(c r)}
$$

But computing the maximum of the function on the right hand side in the given region (e.g. with Maple) shows that for $n>5.5 \cdot 10^{8}$ this does not hold (in fact, the lower bound for $n$ can be chosen slightly smaller for the lower ranges of $r$ ). Hence the Lemma is proved.

For later reference we state the following consequence of the Lemma above:

Corollary 3.6. In the range $5 \leq k \leq 21$, any product of $k$ consecutive integers larger than $5.5 \cdot 10^{8}$ has a prime divisor $q$ bounded from below as follows:

\begin{tabular}{l|rrrrrrrrrrrrrrrrr}
$k=$ & 5 & 6 & 7 & 8 & 9 & 10 & 11 & 12 & 13 & 14 & 15 & 16 & 17 & 18 & 19 & 20 & 21 \\
\hline$q \geq$ & 13 & 17 & 17 & 19 & 23 & 23 & 31 & 37 & 37 & 41 & 41 & 43 & 47 & 53 & 53 & 53 & 59
\end{tabular}

\section{Proof of the main result}

Because of Proposition 2.4 we always assume in this section:

\section{$\lambda=\left(\lambda_{1}, \ldots, \lambda_{l}\right) \in D(n)$ is not a bar, i.e., $l \geq 3$.}

Also, the Theorem is easily checked by hand for $n \leq 28$, so we may assume that $n \geq 29$ when needed.

We use the following notations:

$b_{i}, 1 \leq i \leq \lambda_{1}$ are the first row bar lengths of $\lambda$; note that $b_{i}=b_{1, i+1}=\lambda_{1}+\lambda_{i+1}$ for $i=1, \ldots, l-1$.

$k:=b_{1}-b_{2}-1=\lambda_{2}-\lambda_{3}-1$.

$\mu:=\left(\lambda_{2}, \ldots, \lambda_{l}\right)$, with $b_{1}^{\mu}=b_{2,3}=\lambda_{2}+\lambda_{3}, b_{2}^{\mu}=b_{2,4}=\lambda_{2}+\lambda_{4}$ the two largest first row bar lengths of $\mu$ (where $\lambda_{4}=0$ if $l=3$ ).

$d:=n-b_{1}=\sum_{j \geq 3} \lambda_{j}=\sum_{j \geq 2} \mu_{i}$; note that since we are assuming that $\lambda$ is not a bar, $d>0$.

Lemma 4.1. We have

(1) $|\mu|=\lambda_{3}+k+1+d$.

(2) $|\mu| \leq 2 d+k+1$.

(3) $b_{1}^{\mu}=2 \lambda_{3}+k+1 \leq 2 d+k+1$.

(4) $b_{2}^{\mu}=\lambda_{3}+\lambda_{4}+k+1 \leq d+k+1$.

(5) $b_{2} \geq k+4$.

Proof: (1) follows from the definitions, and then (2) follows from (1). (3),

(4) follow also easily from the definitions.

(5) $b_{2}-k=\lambda_{1}+\lambda_{3}-\lambda_{2}+\lambda_{3}+1=\left(\lambda_{1}-\lambda_{2}\right)+2 \lambda_{3}+1 \geq 4$. 
In addition to the assumption that $\lambda$ is not a bar, we now make the assumption

\section{$\lambda$ is of maximal $\bar{p}$-weight for all odd primes $p \leq n$.}

We define the products

$\pi_{1}:=\left(b_{1}+1\right)\left(b_{1}+2\right) \cdots n, \pi_{i}:=\left(b_{i}+1\right)\left(b_{i}+2\right) \cdots\left(b_{i-1}-1\right)$ for $i=2$ and $i=3$,

having $d, k$ and $\lambda_{3}-\lambda_{4}-1$ consecutive factors, respectively. By definition, the factors defining the products $\pi_{1}, \pi_{2}$ and $\pi_{3}$ are "missing" first row bar lengths of $\lambda$. Thus Proposition 2.2 implies

Lemma 4.2. If $p$ is an odd prime divisor of $\pi_{i}, i=1,2,3$, then $\mu$ has a $p$-bar.

Lemma 4.3. We have

(1) $k \leq 4 \lambda_{3}+1 \leq 4 d+1$.

(2) Let $n>5.5 \cdot 10^{8}$.

If $5 \leq k \leq 10$, then $k \leq \frac{7}{4} \lambda_{3}+1 \leq \frac{7}{4} d+1$.

If $11 \leq k \leq 21$, then $k \leq \frac{4}{3} \lambda_{3}+1 \leq \frac{4}{3} d+1$.

If $22 \leq k \leq 1000$, then $k \leq \lambda_{3} \leq d$.

Proof: (1) For $k \leq 1$, there is nothing to prove. Now assume $k>1$. Since $b_{2} \geq k+4$, Theorem 2.3 shows that one of the $k$ factors in $\pi_{2}$ has a prime divisor $q>\frac{3}{2} k$, or we are in one of the listed exceptional cases. We show first that none of these can occur.

(i) $k=2, b_{2}=2$ or $k=5, b_{2}=5$ are obviously impossible.

(ii) $k=2, b_{2}=7$. Then $\lambda=(6,4,1)$, but this is not of maximal $\overline{3}$-weight, giving a contradiction.

Now let $q$ be a prime divisor of $\pi_{2}$; then there has to be a $q$-bar in $\mu$. Thus, using Lemma 4.1(3) we obtain $\frac{3}{2} k<q \leq b_{1}^{\mu}=2 \lambda_{3}+k+1$, implying $\frac{1}{2} k<2 \lambda_{3}+1$ and thus $k \leq 4 \lambda_{3}+1 \leq 4 d+1$.

For (2), follow the same lines as in (1), but use Lemma 3.5.

We now want to show that we can reduce to small $d$ in all cases, i.e., we want to prove

Proposition 4.4. Let $n \in \mathbb{N}, n \geq 29$. For $\lambda \in D(n)$ with the assumptions as above, we have $d=n-b_{1} \leq 4$.

Proof: By Corollary 3.4(i), we know that for $n \leq 9.25 \cdot 10^{8}$ we have $d \leq 4$, and thus we may now assume that $n>9.25 \cdot 10^{8}$. Then, by Corollary 3.4(ii), we have at least $d \leq 225$. Thus we can now assume that $5 \leq d \leq 225$, and we want to arrive at a contradiction.

Now, Lemma 4.3(1) gives us a bound also for $k$, namely $k \leq 4 d+1 \leq 901$. But then, as $n>5.5 \cdot 10^{8}$, we get an even better bound for $k$ by Lemma 4.3(2), namely $k \leq 225$. Note also that $\lambda_{3}-\lambda_{4}-1<d \leq 225$. 
As $n$ is sufficiently large, we can employ Lemma 3.5 for the $d$ factors in $\pi_{1}$, as well as for the $k$ factors in $\pi_{2}$ when $k \geq 5$, and for the $\lambda_{3}-\lambda_{4}-1$ factors in $\pi_{3}$ when $\lambda_{3}-\lambda_{4}-1 \geq 5$.

Case $22 \leq d \leq 225$. In this situation, $\pi_{1}$ has a prime divisor $q_{1}>3 d$ by Lemma 3.5. Hence $\mu$ has a bar of length divisible by $q_{1}$ in its first row, and $3 d<q_{1} \leq b_{1}^{\mu} \leq$ $2 d+k+1$ (by 4.1), implying $d \leq k$. But then Lemma 4.3(2) implies $k=d$.

Now $\pi_{2}$ has a prime divisor $q_{2}>3 k=3 d$. Thus $\mu$ also has a bar divisible by $q_{2}$ in its first row. If $q_{1}=q_{2}$, then $\mu$ has to have two bars divisible by $q_{1}$ in its first row. Then we obtain $3 d+2 \leq b_{1}^{\mu} \leq 3 d+1$, and thus a contradiction.

We now work downwards to exclude all cases $5 \leq d \leq 21$ as these get slightly more involved for the cases of small $d$. Because of Lemma 4.3 we know in this situation that $k \leq 21$. Then Lemma 4.1 yields

$b_{1}^{\mu}=2 \lambda_{3}+k+1 \leq 2 d+k+1 \leq 64, \quad b_{2}^{\mu}=\lambda_{3}+\lambda_{4}+k+1 \leq d+k+1 \leq 43$.

In particular, for any prime divisor $q$ in the "missing" first row bar lengths we have $q \leq b_{1}^{\mu} \leq 61$.

Case $17 \leq d \leq 21$. Then by Corollary 3.6 there is a prime divisor $q_{1}>43 \geq b_{2}^{\mu}$ in $\pi_{1}$. Thus we must have $q_{1}=b_{1}^{\mu}$. Thus, if $q_{2}$ is a prime divisor in $\pi_{2}$, then we must have $q_{2} \leq b_{2}^{\mu} \leq 43$; using Corollary 3.6 again, this implies $k \leq 16$.

Here and in the following, $q_{1}, q_{2}$ will always be the largest prime divisors of $\pi_{1}, \pi_{2}$, respectively.

If $d=21$, then $q_{1} \geq 59$, and hence $59=b_{1}^{\mu} \leq 2 d+k+1=k+43 \leq 59$, thus $k=16$. Then $q_{2} \geq 43$ in $\pi_{2}$, and then we obtain $q_{2}=43=b_{2}^{\mu} \leq d+k+1=38$, a contradiction. If $d=20$, we have $q_{1} \geq 53$ in $\pi_{1}$, and then $53=b_{1}^{\mu} \leq k+41$ yields $k \geq 12$. Then $q_{2} \geq 37$ in $\pi_{2}$, and thus $37 \leq q_{2} \leq b_{2}^{\mu} \leq k+21$, so even $k \geq 16$. But then we even have $q_{2} \geq 43$ in $\pi_{2}$, giving a contradiction as above. Similarly, for $d=19$, an inequality $53 \leq k+39$ leads to $k \geq 14$, and then to $41 \leq q_{2} \leq b_{2}^{\mu} \leq k+20$, which implies $k=21$, and this yields the contradiction $b_{2}^{\mu} \geq 59$. In the case $d=18$ we get a contradiction quickly, as in the case $d=21$.

If $d=17,47 \leq q_{1} \leq k+35$ leads to $k \geq 12$, hence $37 \leq q_{2} \leq k+d+1 \leq 39$; thus $q_{2}=37$. But this implies both $k \geq 16$ and $k \leq 13$, a contradiction.

Case $d=16$. Then $q_{1} \geq 43, b_{1}^{\mu} \leq 54, b_{2}^{\mu} \leq 38$, hence $q_{1}=b_{1}^{\mu} \leq k+33$ and we must have $10 \leq k \leq 13$. Then $b_{2}^{\mu} \leq 30$ and we obtain $k=10$. Now $q_{1}=b_{1}^{\mu}=43=$ $2 \lambda_{3}+k+1=2 \lambda_{3}+11$, hence $\lambda_{3}=16=d$, i.e., $\lambda_{4}=0$. Now we also consider $\pi_{3}$, which has $\lambda_{3}-1=15$ consecutive factors (which are still sufficiently large) and hence has a prime divisor $q_{3} \geq 41$, giving a contradiction.

Here and in the following, we keep the notation $q_{1}, q_{2}, q_{3}$ for the largest prime divisors of $\pi_{1}, \pi_{2}, \pi_{3}$, respectively.

Case $d=15$. Then by Corollary $3.6 q_{1} \geq 41$. Now $41 \leq b_{1}^{\mu}=2 \lambda_{3}+k+1 \leq 2 d+$ $k+1=k+31$, and thus $k \geq 10$; note also that $k \leq 21$ and thus $b_{1}^{\mu} \leq 52$. If $k \geq 11$, then $q_{2} \geq 31$ and $31 \leq b_{2}^{\mu} \leq d+k+1 \leq 37$; but then we must have $k \leq 13$, and we obtain $b_{2}^{\mu} \leq 29-$ a contradiction. Now it remains to discuss the case $k=10$. Then $41 \leq q_{1} \leq b_{1}^{\mu}=2 \lambda_{3}+k+1 \leq 2 d+k+1=41$, hence $b_{1}^{\mu}=q_{1}=41$ and $\lambda_{3}=d$, i.e., $\lambda_{4}=0$. But now we also have $q_{3} \geq 41$, implying a contradiction. 
Case $d=14$. Here again, $q_{1} \geq 41$, and this yields $k \geq 12$; also, $b_{1}^{\mu} \leq 50$. Now $q_{2} \geq 37$ and thus $37 \leq b_{2}^{\mu} \leq d+k+1 \leq 36$, a contradiction.

Case $d=13$. Here $q_{1} \geq 37$, and thus $k \geq 37-(2 d-1)=10$; also $b_{1}^{\mu} \leq 2 d+k+$ $1 \leq 48$. If $k \geq 11$, then $q_{2} \geq 31$. Then $31 \leq b_{2}^{\mu} \leq d+k+1=35$, and we deduce $q_{2}=31$. Then $k=11$ and we have $b_{2}^{\mu} \leq 25$, a contradiction. Now assume that $k=$ 10. Then $b_{1}^{\mu} \leq 37$, hence $q_{1}=37=b_{1}^{\mu}$ and $\lambda_{3}=d$. Now, $q_{3} \geq 37$, and this gives a contradiction.

Case $d=12$. Here again $q_{1} \geq 37$, and thus $k \geq 12$, and so also $q_{2} \geq 37$. But $b_{1}^{\mu} \leq 46$ and $b_{2}^{\mu} \leq 34$, hence this case is impossible.

Case $d=11$. Here $q_{1} \geq 31$, and then $k \geq 8$. Also $b_{1}^{\mu} \leq 44$, and thus (using $q_{2}$ ) $k \leq 16$. But then $b_{1}^{\mu} \leq 39$ and we must have $k \leq 13$; now $b_{1}^{\mu} \leq 36$, and then even $k \leq 11$, and $q_{1}=31$. Now $b_{2}^{\mu} \leq 23$, hence $k \leq 10$, and then even $b_{2}^{\mu} \leq 22$, and now we must have $k=8$. Then $b_{1}^{\mu}=31$ and $\lambda_{3}=d$, hence $q_{3} \geq 23-$ a contradiction.

Case $d=10$. Here $q_{1} \geq 23$, and then $k \geq 2$. Also $b_{1}^{\mu} \leq 42$, and then $k \leq 15$ and $q_{1} \leq 41$. As $k \leq 15, b_{1}^{\mu} \leq 36$, and then $k \leq 11, q_{1} \leq 31$. This leads to $b_{1}^{\mu} \leq 32$ and $b_{2}^{\mu} \leq 22$, and now we can deduce $q_{1}=b_{1}^{\mu}$ and $k \leq 8$. This gives $q_{1}=b_{1}^{\mu} \leq 29$ and $b_{2}^{\mu} \leq 19$. If $29=q_{1}$, then $q_{1}=b_{1}^{\mu}=2 \lambda_{3}+k+1 \leq 29=2 d+k+1$ implies $k=8$ and $\lambda_{3}=d$. But then $19=q_{2}=b_{2}^{\mu}$ and $q_{3} \geq 23$, giving a contradiction. Hence $q_{1}=$ 23. Note that then $23=q_{1}=b_{1}^{\mu}=2 \lambda_{3}+k+1$ implies that $k$ is even.

Now we have to discuss the cases $k \in\{2,4,6,8\}$. Let $k=8$. Then $23=2 \lambda_{3}+9$, so $\lambda_{3}=7$. Furthermore, $q_{2} \geq 19$, and hence $19=q_{2}=b_{2}^{\mu}=\lambda_{3}+\lambda_{4}+9$, so $\lambda_{3}+\lambda_{4}=$ $10=d$ and $l=4$. Then $b_{3}^{\mu}=\lambda_{2}=\lambda_{3}+1+k=16, b_{4}^{\mu}=15, b_{5}^{\mu}=14, b_{6}^{\mu}=12$ are the next largest bars in $\mu$. But considering the first five and last five consecutive factors in $\pi_{1}$ separately, we see that $\pi_{1}$ has a further prime divisor $q_{4} \geq 13$, giving a contradiction.

Now assume $k=6$. Then $23=b_{1}^{\mu}=2 \lambda_{3}+7$ implies $\lambda_{3}=8$, and then we must have $\lambda_{4}=2, l=4$ and $b_{2}^{\mu}=17=q_{2}$. Now note that $b_{3}^{\mu}=\lambda_{2}=\lambda_{3}+1+k=15$, $b_{4}^{\mu}=14, b_{5}^{\mu}=12$ are the next largest bars in $\mu$. But as $\lambda_{3}-\lambda_{4}-1=5, q_{3} \geq 13$, giving a contradiction.

When $k \leq 4, \lambda_{3} \geq 9$ and then $\lambda_{3}+\lambda_{4}=10=d$ and $b_{2}^{\mu} \leq 15$. But then $q_{3} \geq 17$ gives a contradiction.

Case $d=9$. Again, $q_{1} \geq 23$, and here this implies $k \geq 4$. Also $b_{1}^{\mu} \leq 40$, and then $k \leq$ 13 and $q_{1} \leq 37$. Then, $b_{1}^{\mu} \leq 32$, and thus $k \leq 11$; hence $b_{1}^{\mu} \leq 30$, and thus $k \leq 10$ and we obtain $b_{1}^{\mu} \leq 29$. Moreover, $b_{2}^{\mu} \leq 20$ and now we can deduce $q_{1}=b_{1}^{\mu} \in\{23,29\}$ and $k \leq 8$. This leads to $b_{1}^{\mu} \leq 27$ and hence $q_{1}=b_{1}^{\mu}=23$. As $b_{1}^{\mu}=2 \lambda_{3}+k+1, k$ must be even. As $b_{2}^{\mu} \leq 18$, this yields $k \in\{4,6\}$. If $k=6$, then $b_{2}^{\mu}=16$, giving a contradiction; hence $k=4$. Then $\lambda_{3}=9=d$, i.e., $\lambda_{4}=0$, and thus $q_{3} \geq 18$, giving a contradiction.

Case $d=8$. Here, $q_{1} \geq 19$, and thus $k \geq 2$. Also $b_{1}^{\mu} \leq 38$, and thus $k \leq 13$ and $q_{1} \leq 37$. Then, $b_{1}^{\mu} \leq 30$, and thus $k \leq 10$; hence $b_{1}^{\mu} \leq 27$ and $q_{1} \in\{19,23\}$, and also $b_{2}^{\mu} \leq 19$. If $q_{1}=23$, then also $23=b_{1}^{\mu}=2 \lambda_{3}+k+1$, and thus $k \in\{6,8\}$. But then $b_{2}^{\mu} \leq 17$, hence $k=6$, which gives $b_{2}^{\mu}=15$ and then a contradiction.

It remains to consider the case $q_{1}=19$. Then either $b_{1}^{\mu}=19$ or $b_{2}^{\mu}=19$. First assume $b_{1}^{\mu}=19$; then $k$ is even and $k \leq 6$. Now $b_{2}^{\mu} \leq 15$, hence $k \leq 4$ and then $b_{2}^{\mu} \leq 13$. Then $\lambda_{3} \geq 7$, hence $\lambda_{3}-\lambda_{4}-1 \geq 5$ and thus $q_{3}=13=b_{2}^{\mu}$, and hence 
$k=4, \lambda_{3}=7$. But if $13 \mid \pi_{3}=\prod_{j=14}^{18}(n-j)$, then also $13\left|\prod_{j=1}^{5}(n-j)\right| \pi_{1}=$ $\prod_{j=0}^{7}(n-j)$, and thus we have to have a further 13-bar in $\mu$, a contradiction.

Now we assume $b_{2}^{\mu}=19$; then $k=10$ and $q_{2}=23=b_{1}^{\mu}$, hence $\lambda_{3}=6, \lambda_{4}=2$. Let's take a closer look at the bar lengths in $\mu=(17,6,2)$ :

$\begin{array}{ccccccccccccccccc}23 & 19 & 17 & 16 & 14 & 13 & 12 & 10 & 9 & 8 & 7 & 6 & 5 & 4 & 3 & 2 & 1 \\ & 8 & 6 & 5 & 3 & 2 & 1 & & & & & & & & & & \\ & 2 & 1 & & & & & & & & & & & & & & \end{array}$

Now there are only 15 instances of an odd prime dividing a bar length in $\mu$; hence at least 10 of the "missing" first row bar lengths have to be 2-powers. But the 25 missing first row bar lengths are in the range $n, n-1, \ldots, n-42$, so this is impossible.

Case $d=7$. Here, $q_{1} \geq 17$, and thus $k \geq 2$. Also $b_{1}^{\mu} \leq 36$, and thus $k \leq 11$. Then, $b_{1}^{\mu} \leq 26$, and thus $k \leq 10$; hence $b_{1}^{\mu} \leq 25$ and $q_{1} \in\{17,19,23\}$, and also $b_{2}^{\mu} \leq 18$.

If $q_{1}=23$, then $23=b_{1}^{\mu} \leq 2 d+\bar{k}+1$, and thus $k \geq 8$; but then $q_{2} \leq 19$, which gives a contradiction.

If $q_{1}=19$, then $19=b_{1}^{\mu}=2 \lambda_{3}+k+1$, and thus $k \geq 4$ is even; also, $k \leq 6$ because of $b_{2}^{\mu} \leq 18$. If $k=6$, then $17 \leq q_{2} \leq b_{2}^{\mu} \leq d+k+1=14$, a contradiction. Hence $k=4$, and this implies $\lambda_{3}=7=d$; but then $17 \leq q_{3} \leq b_{2}^{\mu}=12$, a contradiction.

Hence it only remains to consider the case $q_{1}=17$. Here $q_{1}$ can be one of $b_{1}^{\mu}$, $b_{2}^{\mu}$ or $b_{3}^{\mu}$. If $q_{1}=b_{1}^{\mu}=17=2 \lambda_{3}+k+1$, then $b_{2}^{\mu}<17$ and we deduce $k \in\{2,4\}$. If $k=4$, then $\lambda_{3}=6, \lambda_{4}=1$. We take again a look at the bar lengths in $\mu=(11,6,1)$ :

$\begin{array}{ccccccccccc}17 & 12 & 11 & 9 & 8 & 7 & 6 & 4 & 3 & 2 & 1 \\ 7 & 6 & 4 & 3 & 2 & 1 & & & & \end{array}$

There are only 10 instances of an odd prime dividing a bar length in $\mu$; hence at least 8 of the "missing" first row bar lengths have to be 2-powers. But the 18 missing first row bar lengths are in the range $n, n-1, \ldots, n-29$, so this is impossible.

If $k=2$, then $\lambda_{3}=7=d$, hence $q_{3} \geq 17$, giving immediately a contradiction.

If $q_{1}=b_{2}^{\mu}=17=\lambda_{3}+\lambda_{4}+k+1 \leq d+k+1$, then $k \geq 9$ and hence $23 \leq q_{2} \leq$ $b_{1}^{\mu} \leq 25$, so $q_{2}=23=b_{1}^{\mu}=2 \lambda_{3}+k+1$. This implies $k=10, \lambda_{3}=6, \lambda_{4}=1$. But then $b_{2}^{\mu}=\lambda_{3}+\lambda_{4}+k+1=18$, a contradiction.

Finally, assume $q_{1}=b_{3}^{\mu}=17$; then we must have $b_{2}^{\mu}=18$ and thus $k=10$. Then $q_{2} \geq 23$, hence $23=b_{1}^{\mu}=2 \lambda_{3}+k+1$, so $\lambda_{3}=6, \lambda_{4}=1$. Looking at the bar lengths in $\mu=(17,6,1)$, we see that there are 17 instances of an odd prime dividing a bar length in $\mu$ (note that the bar length 15 gives two contributions); hence at least 7 of the "missing" first row bar lengths have to be 2-powers. But the 24 missing first row bar lengths are in the range $n, n-1, \ldots, n-41$, so this is impossible.

Case $d=6$. Again, $q_{1} \geq 17$, and then $k \geq 4$. Also $b_{1}^{\mu} \leq 34$, and thus $k \leq 11$. Then, $b_{1}^{\mu} \leq 24$, and thus $k \leq 10$; hence $b_{1}^{\mu} \leq 23$ and again $q_{1} \in\{17,19,23\}$, and here $b_{2}^{\mu} \leq$ 17.

If $q_{1}=23$, then $b_{1}^{\mu}=q_{1}=23$ and thus $k=10$; but then $q_{2} \geq 23$, giving a contradiction. 
If $q_{1}=19$, then $b_{1}^{\mu}=q_{1}=19$ and this yields $k=6$. Then $\lambda_{3}=6=d$ and $q_{2}=$ $17=b_{2}^{\mu}$, but this leads to $b_{2}^{\mu} \leq d+k+1=13$, a contradiction.

Finally, $q_{1}=17$ and then one of $b_{1}^{\mu}, b_{2}^{\mu}$ equals $q_{1}=17$. If $b_{1}^{\mu}=17$, then we obtain $k=4$ and thus $\lambda_{3}=6=d$; this implies $b_{2}^{\mu}=11$, and hence $q_{3} \geq 13$ gives a contradiction.

If $b_{2}^{\mu}=17$, then $k=10$ and thus $23 \leq q_{2}$, so $q_{2}=23=b_{1}^{\mu}$; this implies $\lambda_{3}=6=$ $d$. Looking again at the bar lengths in $\mu=(17,6)$, we see that there are 16 instances of an odd prime dividing a bar length in $\mu$, hence at least 7 of the "missing" first row bar lengths have to be 2-powers; but the 23 missing first row bar lengths are in the range $n, n-1, \ldots, n-40$, so this is impossible.

Case $d=5$. Here, $q_{1} \geq 13$, and then $k \geq 2$. Also $b_{1}^{\mu} \leq 32$, and thus $k \leq 11$. Then, $b_{1}^{\mu} \leq 22$, and thus $k \leq 8$; hence $b_{1}^{\mu} \leq 19$ and now $q_{1} \in\{13,17,19\}$, and $b_{2}^{\mu} \leq 14$.

If $q_{1}=19$, then $b_{1}^{\bar{\mu}}=q_{1}=19$ and thus $k=8$; but then $q_{2} \geq 19$, giving a contradiction.

If $q_{1}=17$, then $b_{1}^{\mu}=q_{1}=6$ and this implies $k=6$; but then $q_{2} \geq 17$ gives a contradiction.

Finally, $q_{1}=13$ and then one of $b_{1}^{\mu}, b_{2}^{\mu}, b_{3}^{\mu}$ equals $q_{1}=13$. If $b_{1}^{\mu}=13$, then $k=4$ or $k=2$. If $k=4$, then $\lambda_{3}=4$ and $\lambda_{4}=1$. In this case we find 7 instances of an odd prime dividing a bar length in $\mu=(9,4,1)$, hence at least 7 of the "missing" first row bar lengths have to be 2-powers; but the 14 missing first row bar lengths are in the range $n, n-1, \ldots, n-23$, a contradiction.

If $k=2$, then $\lambda_{3}=5=d$. Here, we find 6 instances of an odd prime dividing a bar length in $\mu=(8,5)$, hence at least 7 of the "missing" first row bar lengths have to be 2powers; but the 13 missing first row bar lengths are in the range $n, n-1, \ldots, n-21$, and this is impossible.

If $b_{2}^{\mu}=13$, then $k \geq 7$ and thus $17 \leq q_{2}=b_{1}^{\mu}$; this implies that $k$ is even, so $k=8$ and then $19=q_{2}=b_{1}^{\mu}$, so $\lambda_{3}=5=d$. But then $b_{2}^{\mu}=14$, a contradiction.

Finally we assume $b_{3}^{\mu}=13$; then $b_{2}^{\mu}=14$ and hence $k=8$. Now $q_{2} \geq 19$, hence $q_{2}=b_{1}^{\mu}=19$ and thus $\lambda_{3}=5=d$. Here, there are 12 instances of an odd prime dividing a bar length in $\mu=(14,5)$, hence at least 7 of the "missing" first row bar lengths have to be 2-powers; but the 19 missing first row bar lengths are in the range $n, n-1, \ldots, n-33$, and again this is impossible.

With this final contradiction we have now finished the proof of Proposition 4.4.

\section{Completing the proof of Theorem 1.1}

Because of Proposition 4.4 it only remains to discuss the situation where $d \leq 4$.

Case $d=1$. By Lemma 4.3, we have $k \leq 4 d+1=5$ (without assumption on $n$ ).

Recall that $d=1$ means $\lambda=\left(\lambda_{1}, \lambda_{2}, 1\right), b_{1}=n-1, b_{2}=\lambda_{1}+1, \lambda_{2}=k+2$.

Furthermore, we have the missing first row bar lengths $\pi_{1}=n$ and $b_{2}+1, \ldots, b_{1}-$ $1=\lambda_{1}+2, \ldots, n-2$, when $k>0$.

When $k=0, \mu=(2,1)$, which has the bar lengths $3,1^{2}$, so two missing first row bar lengths have to be 2-powers; note also that $n \geq 6$. We consider the missing first row bar lengths $n$ and $n-5, n-4$ in $\lambda$. But then we can only have $n=6$ or $n=8$. 
For $n=6$, we obtain the partition $(3,2,1)$, for $n=8$, the partition $\lambda=(5,2,1)$ which are both on our list of exceptional bar partitions in Theorem 1.1.

When $k=1, \mu=(3,1)$, which has the bar lengths $4,3,1^{2}$; note that $n \geq 8$. Thus only one missing first row bar length of $\lambda$ can have an odd prime divisor (namely 3 ), and all others have to be 2-powers. But the largest missing first row bar lengths are $n$, $n-2$ and $n-5$, and these can not satisfy this condition.

When $k=2, \mu=(4,1)$, with bar lengths $5,4,2,1^{2}$, so again only one missing first row bar length of $\lambda$ can have an odd prime divisor (here, 5), and all others have to be 2-powers. Here, $n \geq 9$ and we consider $n$ and $n-3, n-2$. But we notice that no two of the three numbers $n, n-2, n-3$ can be simultaneously 2-powers.

When $k=3, \mu=(5,1)$, with bar lengths $6,5,3,2,1^{2}$, so two missing first row bar lengths of $\lambda$ can have an odd prime divisor 3 and one missing first row bar length has the prime divisor 5, and all others have to be 2-powers, in particular, at least three missing first row bar lengths have to be 2-powers. Here, $n \geq 11$ and we consider the missing first row bar lengths $n, n-4, n-3, n-2$ and $n-7$. None of $n, n-2, n-4$ can be 2-powers, as then none of the other four numbers can be a 2-power; thus, $n-3$ and $n-7$ have to be 2-powers, implying $n=11$, which gives a contradiction.

When $k=4, \mu=(6,1)$, with bar lengths $7,6,4,3,2,1^{2}$, so two missing first row bar lengths of $\lambda$ can have an odd prime divisor 3 and one missing first row bar length has the prime divisor 7, and all others have to be 2-powers, in particular, at least four missing first row bar lengths have to be 2-powers. Here, $n \geq 13$ and we consider the missing first row bar lengths $n, n-2, n-3, n-4, n-5$ and $n-8$. But it is impossible that three of these numbers are 2-powers.

When $k=5, \mu=(7,1)$, with bar lengths $8,7,5,4,3,2,1^{2}$, so at least five missing first row bar lengths have to be 2-powers. Here, $n \geq 15$ and we consider the missing first row bar lengths $n, n-2, n-3, n-4, n-5, n-6$. But it is impossible that three of these numbers are 2-powers.

Case $d=2$. By Lemma 4.3 we have $k \leq 4 d+1=9$.

Recall that $d=2$ means $\lambda=\left(\lambda_{1}, \lambda_{2}, 2\right), b_{1}=n-2, b_{2}=\lambda_{1}+2, \lambda_{2}=k+3$.

Furthermore, we have the missing first row bar lengths $n, n-1$ and $b_{2}+$ $1, \ldots, b_{1}-1=n-2-k, \ldots, n-3, n-4-k, n-7-k$ and $n-9-k$ when $k>0$. Note that if $k$ is odd, then we have $(k+5) / 2$ each of missing first row bar lengths congruent to $n$ and $n-1 \bmod 2$, respectively, if $k>0$ is even, then we have $k / 2+2$ and $k / 2+3$ missing first row bar lengths congruent to $n$ and $n-1 \bmod 2$, respectively.

For $k=0, \mu=(3,2)$ with bar lengths $5,3,2^{2}, 1$, so at least three of the missing first row bar lengths $n, n-1, n-4, n-3, n-8$ are 2-powers. But this is only possible if $n=9$, and this gives the bar partition $\lambda=(4,3,2)$ which is on our list of exceptional cases.

For $1 \leq k \leq 9$, the number of 2-powers among the missing first row bar lengths forced by the 2-power bar lengths in $\mu$ as in the previous arguments, quickly shows in each case that no further exceptional case occurs. (Note that one might also use Hanson's result to exclude some cases, but the arguments then take slightly longer and are a bit more involved.) 
Case $d=3$. Here Lemma 4.3 yields $k \leq 4 d+1=13$. For the discussion of this case, again we do not assume that $n \geq 29$. In this situation, we have two types of bar partitions to discuss, namely type I: $\lambda=\left(\lambda_{1}, \lambda_{2}, 3\right)$ and type II: $\lambda=\left(\lambda_{1}, \lambda_{2}, 2,1\right)$.

Type I: $\lambda=\left(\lambda_{1}, \lambda_{2}, 3\right)$. Again, we consider the missing first row bar lengths; here these are $n, n-1, n-2 ; n-4, \ldots, n-3-k$ (when $k>0) n-5-k, n-6-k$; $n-10-k$ and $n-11-2 k$. Note that $n \geq 12+2 k$.

When $k=0$, the bar lengths in $\mu$ are (with multiplicities) $7,4,3^{2}, 2^{2}, 1$, in particular, only one missing first row bar length has a prime divisor $>3$ and none has a prime divisor $>7$. Using Hanson's Theorem for the sequences $n, n-1, n-2$ and $n-5, n-6$, we see that this is not possible.

When $k=1$, the bar lengths in $\mu$ are $8,5,4,3^{2}, 2,1$, and a similar reasoning on $n, n-1, n-2$ and $n-6, n-7$ gives a contradiction.

For $k=2$, the bar lengths in $\mu$ are 9, 6, 5, 4, 3, $2^{2}, 1^{2}$, and by Hanson's Theorem used on $n, n-1, n-2$ and $n-4, n-5$ we have a contradiction.

For $k=3$, the bar lengths in $\mu$ are $10,7,6,5,3^{2}, 2^{2}, 1^{2}$, and thus we must have at least four 2-powers among the missing first row bar lengths. But then there must be at least four 2-powers among either the numbers $n, n-2, n-4, n-6, n-8$ or among the numbers $n-1, n-5, n-9, n-13, n-17$, and this is impossible.

The cases $k=4,5,6,7$ can be dealt with in an analogous way.

For $k=8,9,11,13$, Hanson's Theorem gives a large prime divisor in $(n-$ 4) $\cdots(n-3-k)$ which is bigger than the prime divisors in the bar lengths of $\mu$.

For $k=10, \mu$ has the bar lengths $17,14,13,12,10,9,8,7,6,5,4,3^{2}, 2^{2}, 1^{2}$, so we have only 6 bar lengths divisible by a prime $\geq 5$. Now using Hanson's Theorem on $n, n-1, n-2$ gives a prime divisor $\geq 5$, then $(n-4) \ldots(n-13)$ has two different prime divisors $\geq 11$, but it also has a prime divisor 7 and two factors divisible by 5 , and finally $n-15, n-16$ has a further prime divisor $\geq 5$, a contradiction.

For $k=12$, a similar argument is used.

Type II: $\lambda=\left(\lambda_{1}, \lambda_{2}, 2,1\right)$. Here indeed we get the exceptional example $(4,3,2,1)$ for $k=0$. For $k>0$, in a tedious case-by-case analysis using similar arguments as above all cases can be handled and no further exceptional cases occur.

Case $d=4$. Lemma 4.3 gives $k \leq 4 d+1=17$.

Here, we have again two types of bar partitions to discuss, namely type I: $\lambda=$ $\left(\lambda_{1}, \lambda_{2}, 4\right)$ and type II: $\lambda=\left(\lambda_{1}, \lambda_{2}, 3,1\right)$.

As above, a tedious case-by-case analysis using again Hanson's Theorem and sometimes also the requirement of first row bar lengths to be 2-powers allows to deal with all cases, and no new exceptional cases occur.

Thus the Theorem is proved.

Acknowledgment The author would like to thank the Isaac Newton Institute for Mathematical Sciences of the University of Cambridge for its support during a stay there in the frame of the programme Symmetric functions and Macdonald polynomials, where an early part of the work for this article was done.

Thanks go also to Prof. J. Zhang for the hospitality enjoyed during a visit at Peking University when the work for this article was completed; the support of this stay by the Sino-German Center for Research Promotion in Beijing is gratefully acknowledged. 


\section{References}

1. A. Balog, C. Bessenrodt, J.B. Olsson, and K. Ono, "Prime power degree representations of the symmetric and alternating groups," J. London Math. Soc. 64(2) (2001), 344-356.

2. C. Bessenrodt, G. Malle, and J.B. Olsson, "Separating characters by blocks," J. London Math. Soc. 73(2) (2006), 493-505.

3. C. Bessenrodt and J.B. Olsson, "The 2-blocks of the covering groups of the symmetric groups," Advances in Math. 129 (1997), 261-300.

4. C. Bessenrodt and J.B. Olsson, "Prime power degree representations of the double covers of the symmetric and alternating groups," J. London Math. Soc. 66(2) (2002), 313-324.

5. C. Bessenrodt and J.B. Olsson, "Weights of partitions and character zeros," Electron. J. Comb. 11(2) (2004), R5.

6. D. Hanson, "On a theorem of Sylvester and Schur," Canad. Math. Bull. 16 (1973), 195-199.

7. P.N. Hoffman and J.F. Humphreys, Projective Representations of the Symmetric Groups, Clarendon Press Oxford, 1992.

8. G. James and A. Kerber, "The representation theory of the symmetric group," Encyclopedia of Mathematics and its Applications 16, Addison-Wesley (1981).

9. G. Malle, G. Navarro, and J.B. Olsson, "Zeros of characters of finite groups," J. Group Theory 3 (2000), 353-368.

10. A.O. Morris, "The spin representation of the symmetric group," Canad. J. Math. 17 (1965), 543-549.

11. A.O. Morris and A.K. Yaseen, "Some combinatorial results involving Young diagrams," Math. Proc. Camb. Phil. Soc. 99 (1986), 23-31.

12. J.B. Olsson, "Combinatorics and representations of finite groups," Vorlesungen aus dem FB Mathematik der Univ. Essen, Heft 20 (1993).

13. I. Schur, "Einige Sätze über Primzahlen mit Anwendungen auf Irreduzibilitätsfragen. I," Sitzungsberichte der Preuss. Akad. d. Wiss. 1929, Phys.-Math. Klasse, pp. 125-136. 